PROCEEDINGS OF THE

AMERICAN MATHEMATICAL SOCIETY

Volume 131, Number 7 , Pages 2043-2048

S 0002-9939(02)06790-4

Article electronically published on November 13, 2002

\title{
CONSTRAINTS FOR THE NORMALITY OF MONOMIAL SUBRINGS AND BIRATIONALITY
}

\author{
ARON SIMIS AND RAFAEL H. VILLARREAL \\ (Communicated by Wolmer V. Vasconcelos)
}

\begin{abstract}
Let $k$ be a field and let $\mathbf{F} \subset k\left[x_{1}, \ldots, x_{n}\right]$ be a finite set of monomials whose exponents lie on a positive hyperplane. We give necessary conditions for the normality of both the Rees algebra $R[\mathbf{F} t]$ and the subring $k[\mathbf{F}]$. If the monomials in $\mathbb{F}$ have the same degree, one of the consequences is a criterion for the $k$-rational map $F: \mathbb{P}_{k}^{n-1} \rightarrow \mathbb{P}_{k}^{m-1}$ defined by $\mathbf{F}$ to be birational onto its image.
\end{abstract}

\section{A group theoretical CONStraint For normal ReEs AlgEbras}

Let $R=k[\mathbf{x}]=k\left[x_{1}, \ldots, x_{n}\right]$ be a polynomial ring over a field $k$. In the sequel we consider a finite set of monomials $\mathbf{F}=\left\{\mathbf{x}^{\alpha_{1}}, \ldots, \mathbf{x}^{\alpha_{m}}\right\} \subset R$. Associating to a monomial $\mathbf{x}^{\alpha}:=x_{1}^{a_{1}} \cdots x_{n}^{a_{n}}$ its exponent vector $\alpha=\left(a_{1}, \ldots, a_{n}\right) \in \mathbb{N}^{n}$ gives rise to the integer matrices

$$
\mathcal{A}(\mathbf{F})=\left(\alpha_{1}, \ldots, \alpha_{m}\right) \text { and } \mathcal{A}^{\prime}(\mathbf{F})=\left(\begin{array}{ccc}
\alpha_{1} & \cdots & \alpha_{m} \\
1 & \cdots & 1
\end{array}\right),
$$

where the $\alpha_{i}$ 's are regarded as column vectors; $\mathcal{A}(\mathbf{F})$ is sometimes called the logmatrix of $\mathbf{F}$. Note that, given a simple graph, the log-matrix of the squarefree degree two monomials corresponding to its edges is precisely the incidence matrix of the graph.

Assume that the exponents of the monomials belonging to $\mathbf{F}$ lie on a positive hyperplane, that is to say, there exists a vector $x_{0} \in \mathbb{Z}^{n}$ with positive entries such that

$$
\left\langle x_{0}, \alpha_{i}\right\rangle=d \quad(i=1, \ldots, m),
$$

for some $d \geq 2$, where $\left\langle x_{0}, \alpha_{i}\right\rangle$ is the standard inner product of $x_{0}$ and $\alpha_{i}$.

As an extra bit of notation, if $C$ is an $r \times m$ integral matrix, we denote by $\mathbb{Z} C$ the subgroup of $\mathbb{Z}^{r}$ generated by the columns of $C$, while $\Delta_{s}(C)$ will denote the greatest common divisor of all the nonzero $s \times s$ minors of $C$.

Received by the editors September 10, 2001 and, in revised form, March 7, 2002.

2000 Mathematics Subject Classification. Primary 13H10; Secondary 14E05, 14E07, 13B22.

Key words and phrases. Birational map, minors, normal ideal, Rees algebras.

The first author was partially supported by a CNPq grant and PRONEX-ALGA (Brazilian Group in Commutative Algebra and Algebraic Geometry).

The second author was supported in part by CONACyT grant 27931E. This author thanks PRONEX-ALGA for their hospitality. 
Closely associated to $\mathbf{F}$ is the Rees algebra $R[\mathbf{F} t] \subset R[t]$, where $t$ is a new variable independent of the $\mathbf{x}$. It is well-known that the domain $R[\mathbf{F} t]$ is normal if and only if the ideal $(\mathbf{F}) \subset R$ is normal.

The following result seems to have gone unnoticed. It provides an obstruction for the normality of $(\mathbf{F}) \subset R$.

Proposition 1.1. As above, let $\mathbf{F}$ stand for a finite set of monomials and let $x_{0} \in \mathbb{Z}^{n}$ be given with positive entries satisfying $\left\langle x_{0}, \alpha_{i}\right\rangle=d, i=1, \ldots, m$, for some $d \geq 2$. Suppose that the ideal $(\mathbf{F}) \subset R$ is normal. $d$.

(a) Then the torsion subgroup of $\mathbb{Z}^{n} / \mathbb{Z} \mathcal{A}(\mathbf{F})$ is a cyclic group of order dividing

(b) If $\mathbb{Z}^{n} / \mathbb{Z} \mathcal{A}(\mathbf{F})$ is a finite group and $x_{0}=(1, \ldots, 1)$ (i.e., the $\alpha_{i}$ 's have total degree $d)$, then $\mathbb{Z}^{n} / \mathbb{Z} \mathcal{A}(\mathbf{F}) \simeq \mathbb{Z}_{d}$.

Proof. For an abelian group $(M,+)$ its torsion subgroup will be denoted by $T(M)$. It is not hard to see that there is an exact sequence of finite groups

$$
0 \longrightarrow T\left(\mathbb{Z}^{n+1} / \mathbb{Z} \mathcal{A}^{\prime}(\mathbf{F})\right) \stackrel{\varphi}{\longrightarrow} T\left(\mathbb{Z}^{n} / \mathbb{Z} \mathcal{A}(\mathbf{F})\right) \stackrel{\psi}{\longrightarrow} \mathbb{Z}_{d},
$$

where the maps $\varphi$ and $\psi$ are given by

$$
\begin{aligned}
\varphi(\overline{(\alpha, b)}) & =\bar{\alpha} \\
\psi(\bar{\alpha}) & =\overline{\left\langle\alpha, x_{0}\right\rangle} .
\end{aligned} \quad\left(\alpha \in \mathbb{Z}^{n}, \quad b \in \mathbb{Z}\right),
$$

Since $R[\mathbf{F} t]$ is normal, one has $\Delta_{r}\left(\mathcal{A}^{\prime}(\mathbf{F})\right)=1$ (cf. [2]), where $r$ is the rank of $\mathcal{A}^{\prime}(\mathbf{F})$. Since $\Delta_{r}\left(\mathcal{A}^{\prime}(\mathbf{F})\right)$ is the order of $T\left(\mathbb{Z}^{n+1} / \mathbb{Z} \mathcal{A}^{\prime}(\mathbf{F})\right)$, part (a) follows using the exact sequence $(*)$. To prove (b), note that $\mathbb{Z}^{n} / \mathbb{Z} \mathcal{A}(\mathbf{F})$ is a torsion group and the $i$ th unit vector $\bar{e}_{i}$ maps into the element $\overline{1}$ under the map $\psi$. Hence $\psi$ is onto, and $\psi$ is the required isomorphism.

Recall that an extension $A \subset B$ of integral domains is said to be birational if $A$ and $B$ have the same field of fractions. Let

$$
\mathbf{F}=\left\{x^{\alpha_{1}}, \ldots, x^{\alpha_{m}}\right\} \quad \text { and } \quad \mathbf{G}=\left\{x^{\beta_{1}}, \ldots, x^{\beta_{r}}\right\}
$$

be two sets of monomials of $R$ such that $\mathbf{F} \subset \mathbf{G}$. Note that in this situation, the ring extension is birational if and only every monomial of $\mathbf{G}$ can be written as a fraction whose members are suitable power products of the monomials of $\mathbf{F}$. Taking log, it readily follows that $k[\mathbf{F}] \subset k[\mathbf{G}]$ is a birational extension if and only if $\mathbb{Z} \mathcal{A}(\mathbf{F})=\mathbb{Z} \mathcal{A}(\mathbf{G})$.

In the sequel let $\mathbf{x}_{d}$ denote the set of all monomials of degree $d$ in $R$. Then $k\left[\mathbf{x}_{d}\right]$ is the $d t h$ Veronese subring $R^{(d)}$ of $R$.

The following simple criterion for a monomial ring extension to be birational will be used in Section 2. It seems to be floating around, but we could not find an explicit reference for it in the present formulation.

Lemma 1.2. If $\mathbf{F} \subset \mathbf{x}_{d}$, then $k[\mathbf{F}] \subset k\left[\mathbf{x}_{d}\right]$ is a birational extension if and only if $n=\operatorname{rank}(\mathcal{A}(\mathbf{F}))$ and $\Delta_{n}(\mathcal{A}(\mathbf{F}))=d$.

Proof. Assume the given extension is birational. Then $\mathbb{Z} \mathcal{A}\left(\mathbf{x}_{d}\right)=\mathbb{Z} \mathcal{A}(\mathbf{F})$ by the above remark. By Proposition 1.1 (b), or by direct observation, $\mathbb{Z}^{n} / \mathbb{Z} \mathcal{A}\left(\mathbf{x}_{d}\right) \simeq \mathbb{Z}_{d}$. Therefore $\Delta_{n}(\mathcal{A}(\mathbf{F}))=d$.

Conversely, if $\Delta_{n}(\mathcal{A}(\mathbf{F}))=d$, the surjection

$$
\mathbb{Z}^{n} / \mathbb{Z} \mathcal{A}(\mathbf{F}) \longrightarrow \mathbb{Z}_{d}, \quad \bar{a} \longmapsto \overline{|a|}
$$


is an isomorphism. Since $\log \left(\mathbf{x}_{d}\right)$ maps to zero under this map, one obtains $\mathbb{Z} \mathcal{A}(\mathbf{F})=$ $\mathbb{Z} \mathcal{A}\left(\mathbf{x}_{d}\right)$, as required.

\section{BIRATIONALITY CRITERION FOR MONOMIAL RATIONAL MAPS}

Quite generally, any set $\mathbf{F}$ of $m$ forms of the same degree in $k[\mathbf{x}]$ defines a $k$ rational map $F: \mathbb{P}_{k}^{n-1} \rightarrow \mathbb{P}_{k}^{m-1}$. Let $W \subset \mathbb{P}_{k}^{m-1}$ be its image - a unirational variety whose homogeneous coordinate ring is $k[\mathbf{F}] \subset k[\mathbf{x}]$ after regrading $k[\mathbf{F}]$ so that its generators have degree one. Naturally enough, one says that $F$ is birational onto its image if there exists a $k$-rational map $G: W \rightarrow \mathbb{P}_{k}^{n-1}$ which is an inverse of $F$. Classical literature would refer to this situation by saying that $F$ is a rational representation of the projective space $\mathbb{P}_{k}^{n-1}$. The case where $\mathbf{F}$ is a set of monomials of the same degree defining a birational map onto the image would then be called a monomial rational representation (of the projective space $\mathbb{P}_{k}^{n-1}$ ).

Proposition 2.1. Let $\mathbf{F}$ be a set of monomials of the same degree $d \geq 1$ in $k[\mathbf{x}]$ and let $\mathcal{A}(\mathbf{F})$ denote the corresponding log-matrix. Then $\mathbf{F}$ defines a monomial rational representation if and only if $\Delta_{n}(\mathcal{A}(\mathbf{F}))=d$.

Proof. The proof is based on the following observation of general nature: let $A$ and $B$ be standard graded domains over a field $k$, with respective fraction fields $k(A)$ and $k(B)$, and let $A \subset B$ be a homogeneous (i.e., a degree zero) inclusion. Then the corresponding dominant rational map $X=\operatorname{Proj}(B) \rightarrow Y=\operatorname{Proj}(A)$ is birational (if and) only if $k(A)=k(B)$ (cf. [6], where this is used over and again). To see why this is true, notice the well-known fact that if $z \in A$ is any nonzero homogeneous element of degree one, then $k(A)=k(Y)(z)$ and $k(B)=k(Y)(z)$, where $k(X)$ and $k(Y)$ are the respective function fields of $X$ and $Y$.

We now apply this result to our situation with $A=k[\mathbf{F}]$ and $B=k\left[\mathbf{x}_{d}\right]$. This is a homogeneous inclusion of graded rings and we only need to regrade both so as to have them generated in degree one. The result is then that the corresponding rational map between the projective varieties is birational if and only if the inclusion $k[\mathbf{F}] \subset k\left[\mathbf{x}_{d}\right]$ has the same field of fractions. Since $\operatorname{Proj}\left(k\left[\mathbf{x}_{d}\right]\right)$ coincides (after the above regrading) with the $d$ th Veronesean embedding of $\mathbb{P}_{k}^{n-1}=\operatorname{Proj}(k[\mathbf{x}])$, it follows that the $k$-rational map $F: \mathbb{P}_{k}^{n-1} \rightarrow \operatorname{Im}(F) \subset \mathbb{P}_{k}^{m-1}$ is a rational representation if and only if the inclusion $k[\mathbf{F}] \subset k\left[\mathbf{x}_{d}\right]$ has the same field of fractions. Now apply Lemma 1.2,

Remark. The above criterion is known in the affine situation and when $m=n$. Namely, a set of $n$ monomials in $k\left[x_{1}, \ldots, x_{n}\right]$, possibly with negative exponents, defines a birational map of $\mathbb{A}_{k}^{n}$ to itself if and only if the corresponding log-matrix belongs to $\mathrm{SL}(n, k)$, in which case the inverse rational map has as log-matrix the inverse matrix (cf. [4, p. 114, No. 5]).

\section{Normality of the Rees algebra versus birationality}

Here we present some applications. We keep the notation introduced in the previous sections.

Corollary 3.1. Let $\mathbf{F}$ be a set of monomials of degree $d \geq 1$ in $k\left[x_{1}, \ldots, x_{n}\right]$ and let $I=(\mathbf{F})$ be the ideal generated by $\mathbf{F}$. If $\operatorname{rank}(\mathcal{A}(\mathbf{F}))=n$ and $I$ is a normal ideal, then $\mathbf{F}$ defines a monomial rational representation.

Proof. It follows from Propositions 1.1 and 2.1 
Let $G$ be a simple connected graph and let $\mathbf{F}_{\mathbf{G}} \subset k\left[x_{1}, \ldots, x_{n}\right]$ be the set of squarefree monomials of degree 2 corresponding to the edges of $G$.

Corollary 3.2. If $G$ is not bipartite and contains no $H$-configurations, then $\mathbf{F}_{\mathbf{G}}$ defines a monomial rational representation of $\mathbb{P}_{k}^{n-1}$.

Proof. For the notion of an $H$-configuration, and the related notion of a bow tie configuration, we refer to [9, Chapter 8, p. 314] and [7]. Since $\operatorname{rank}\left(\mathcal{A}\left(\mathbf{F}_{\mathbf{G}}\right)\right)=n$ for a non-bipartite graph, the result follows immediately from Corollary 3.1 by using 7, Theorem 1.1 and Corollary 2.8] which together assert that the edge ideal $\left(\mathbf{F}_{\mathbf{G}}\right)$ is normal if and only if the graph $G$ admits no $H$-configurations.

The present method gives a fresh view of a conjecture of [5. Conjecture 2.8], which we now prove to be true.

Corollary 3.3. If $G$ is not a tree, then the following conditions are equivalent:

(i) The ideal $\left(\mathbf{F}_{\mathbf{G}}\right)$ is of linear type.

(ii) $G$ has exactly one cycle and this cycle is odd.

(iii) $\mathbf{F}_{\mathbf{G}}$ defines a Cremona transformation of $\mathbb{P}_{k}^{n-1}$.

Proof. As observed in [5], only implication (ii) $\Rightarrow$ (iii) was still unproved. But now Corollary 3.2 implies that $\mathbf{F}_{\mathbf{G}}$ defines a monomial rational representation of $\mathbb{P}_{k}^{n-1}$ which, for $m=n$, means that the rational map is a Cremona transformation.

\section{A NECESSARY CONDITION FOR NORMALITY}

In this section the vector $x_{0}$ that makes $k[\mathbf{F}]$ a homogeneous subring is not required to have positive entries.

Let us denote by $P$ the toric ideal of $k[\mathbf{F}]$ which is the kernel of the epimorphism

$$
\varphi: S=k\left[t_{1}, \ldots, t_{m}\right] \longrightarrow k[\mathbf{F}] \quad\left(t_{i} \stackrel{\varphi}{\longmapsto} x^{\alpha_{i}}\right)
$$

of $k$ algebras, where $S$ is a polynomial ring in the $t_{i}$ variables.

A binomial $\mathbf{t}^{\alpha}-\mathbf{t}^{\beta}$ in $S$ is said to have a square-free term if at least one of its two terms $\mathbf{t}^{\alpha}, \mathbf{t}^{\beta}$ is square-free.

The following is a necessary condition for the normality of a homogeneous subring $k[\mathbf{F}]$ in terms of its toric ideal.

Proposition 4.1. Let $k[\mathbf{F}]$ be a homogeneous monomial subring and let $\mathbf{B}$ be a finite set of binomials in the toric ideal $P$ of $k[\mathbf{F}]$. If $k[\mathbf{F}]$ is normal and $P$ is minimally generated by $\mathbf{B}$, then every element of $\mathbf{B}$ has a square-free term.

Proof. Since $k[\mathbf{F}]$ is homogeneous, $P$ is a graded ideal with respect to the standard grading of $S$ induced by $\operatorname{deg}\left(t_{i}\right)=1$. Set $\mathbf{F}=\left\{f_{1}, \ldots, f_{m}\right\}$ and $\mathbf{B}=\left\{g_{1}, \ldots, g_{\ell}\right\}$, where $f_{i}=\mathbf{x}^{\alpha_{i}}$. Let $g$ be a binomial in $\mathbf{B}$. We proceed by contradiction assuming $g$ has no square free-term. After permuting the $t_{i}$ variables one can write

$$
g=t_{1}^{a_{1}} \cdots t_{r}^{a_{r}}-t_{r+1}^{a_{r+1}} \cdots t_{s}^{a_{s}},
$$

with $a_{i} \geq 1$ for all $i$ and

$$
2 \leq a_{1}=\max \left\{a_{1}, \ldots, a_{r}\right\} \leq a_{s}=\max \left\{a_{r+1}, \ldots, a_{s}\right\} .
$$

From the equality

$$
f_{1}^{a_{1}} \cdots f_{r}^{a_{r}}=f_{r+1}^{a_{r+1}} \cdots f_{s}^{a_{s}}
$$


we obtain $f_{1} \cdots f_{r} / f_{s} \in k[\mathbf{F}]$ because $k[\mathbf{F}]$ is normal. Hence there exists a binomial $h_{1}$ in $P$ of the form

$$
h_{1}=t_{1} \cdots t_{r}-t_{s} \mathbf{t}^{\alpha}
$$

with $r=\operatorname{deg}\left(h_{1}\right)=\operatorname{deg}\left(\mathbf{t}^{\alpha}\right)+1<\operatorname{deg}(g)$. Note the equality

$$
g-t_{1}^{a_{1}-1} \cdots t_{r}^{a_{r}-1} h_{1}=t_{s} h_{2},
$$

where $h_{2}$ is a binomial in $P$ with $\operatorname{deg}\left(h_{2}\right)<\operatorname{deg}(g)$ or $h_{2}=0$. Writing

$$
h_{j}=\sum_{g_{i} \neq g} c_{i j} g_{i} \quad(j=1,2)
$$

and using (4.1) we conclude

$$
g=\sum_{g_{i} \neq g} c_{i} g_{i} \quad\left(c_{i} \in S\right)
$$

a contradiction because $P$ is minimally generated by $g_{1}, \ldots, g_{\ell}$.

A full converse to Proposition 4.1 is not true even if the monomials have the same positive total degree, as the following simple example shows. Incidentally, the example gives a monomial rational representation of $\mathbb{P}^{3}$ thus showing that the converse to Corollary [3.1 does not hold either.

Example 4.2. Let $\mathbf{F}=\left\{x_{1} x_{2}, x_{2} x_{3}, x_{3} x_{4}, x_{1} x_{4}, x_{1}^{2}, x_{2}^{2}, x_{3}^{2}, x_{4}^{2}\right\} \subset k\left[x_{1}, x_{2}, x_{3}, x_{4}\right]$. Using a procedure in Macaulay 2 [3], one obtains that $P$ is minimally generated by

$$
\begin{array}{r}
\mathbf{B}=\left\{t_{4}^{2}-t_{5} t_{8}, t_{3}^{2}-t_{7} t_{8}, t_{1} t_{3}-t_{2} t_{4}, t_{2}^{2}-t_{6} t_{7},\right. \\
\left.t_{1}^{2}-t_{5} t_{6}, t_{3} t_{4} t_{6}-t_{1} t_{2} t_{8}, t_{2} t_{3} t_{5}-t_{1} t_{4} t_{7}\right\} .
\end{array}
$$

On the other hand using Normaliz [1] we obtain $\overline{k[\mathbf{F}]}=k[\mathbf{F}]\left[x_{1} x_{3}, x_{2} x_{4}\right]$.

Notice however the following surprising partial converse due to Bernd Sturmfels.

Theorem $4.3([8])$. Let $<$ be a monomial order of $S$. If $k[\mathbf{F}]$ is homogeneous and the initial ideal of $P$ is square-free, then $k[\mathbf{F}]$ is normal.

\section{ACKNOWLEDGMENT}

The first author is indebted to Bernd Ulrich for preliminary discussions leading to the formulation of the criterion given in Proposition 2.1.

\section{REFERENCES}

1. W. Bruns and R. Koch, Normaliz, a program to compute normalizations of affine semigroups. Available from ftp.mathematik. Uni-Osnabrueck.DE/pub/osm/kommalg/software.

2. C. Escobar, J. Martínez-Bernal and R. Villarreal, A comparison of Ehrhart rings with some other monomial subrings, Reporte Técnico No. 303, CINVESTAV-IPN, 2001.

3. D. Grayson and M. Stillman, Macaulay2, 1996. Available via ftp from math.uiuc.edu.

4. W. Gröbner, Algebraische Geometrie, 2. Teil, Bibliographisches Institut Manheim, 1970. MR 48:8499

5. F. Russo and A. Simis, On birational maps and Jacobian matrices, Compositio Math. 126 (2001), 335-358. MR 2002d:14018

6. A. Simis, B. Ulrich and W. V. Vasconcelos, Codimension, multiplicity and integral extensions, Math. Proc. Camb. Phil. Soc. 130 (2001), 237-257. MR 2002c:13017

7. A. Simis, W. V. Vasconcelos and R. Villarreal, The integral closure of subrings associated to graphs, J. Algebra 199 (1998), 281-289. MR 99c:13004 
8. B. Sturmfels, Gröbner Bases and Convex Polytopes, University Lecture Series 8, American Mathematical Society, Rhode Island, 1996. MR 97b:13034

9. R. Villarreal, Monomial Algebras, Monographs and Textbooks in Pure and Applied Mathematics 238, Marcel Dekker, Inc., New York, 2001. MR 2002c:13001

Departamento de Matemática, Universidade Federal de Pernambuco, 50740-540 ReCIFE, Pe, Brazil

E-mail address: aron@dmat.ufpe.br

Departamento de Matemáticas, Centro de Investigación y de Estudios Avanzados del iPN, Apartado Postal 14-740, 07000 México City, D.F., Mexico

E-mail address: vila@esfm.ipn.mx 